\title{
Chiral measurements with the Fixed-Point Dirac operator and construction of chiral currents
}

\author{
P. Hasenfratz ${ }^{\mathrm{a} *}$, S. Hauswirth ${ }^{\mathrm{a}}$, K. Holland ${ }^{\mathrm{a}}$, T. Jörg ${ }^{\mathrm{a}}$, F. Niedermayer ${ }^{\mathrm{a}}$ \\ ${ }^{a}$ Institute for Theoretical Physics, University of Bern, Sidlerstrasse 5, CH-3012 Bern, Switzerland
}

\begin{abstract}
In this preliminary study, we examine the chiral properties of the parametrized Fixed-Point Dirac operator $D^{\mathrm{FP}}$, see how to improve its chirality via the Overlap construction, measure the renormalized quark condensate $\hat{\Sigma}$ and the topological susceptibility $\chi_{t}$, and investigate local chirality of near zero modes of the Dirac operator. We also give a general construction of chiral currents and densities for chiral lattice actions.
\end{abstract}

\section{Introduction}

There has been enormous recent interest in lattice QCD with chiral fermions, with several approaches now being used in simulations [1]. We use one approach, Fixed-Point actions [2], which have many desirable features, including chiral symmetry. This preliminary study gives the first chiral measurements with this action. We also show how to construct chiral densities and conserved currents for chiral actions.

\section{Chiral properties of $D^{\mathrm{FP}}$}

A lattice Dirac operator $D$ which satisfies the Ginsparg-Wilson (GW) relation [3]

$\left\{D^{-1}, \gamma_{5}\right\}=2 a R \gamma_{5}$,

has exact chiral symmetry at non-zero lattice spacing $a$ [4]. The exact Fixed-Point Dirac operator satisfies exactly the GW relation [5] with a non-trivial local function $R$. The function $2 R$ can be absorbed into the definition of $D$ [6] and, for notational simplicity, we shall write $2 R=1$ in most of the following equations. The parametrized Fixed-Point Dirac operator $D^{\mathrm{FP}}$, as described in [7], is an approximate solution of the GW relation. In Fig. 1, we show the eigenvalues of $D^{\mathrm{FP}}$ for Fixed-Point gauge action $S_{g}^{\mathrm{FP}}$ configurations [8] of volume $4^{4}$ and $5^{4}$ with lattice spacing $a \approx 0.16 \mathrm{fm}$ (as measured by the Sommer parameter $r_{0}$ ). For an exact solution $D$ of the GW relation, the complex eigenvalues of $D$

\footnotetext{
*Based on a talk by T.J. and a poster by K.H.
}

lie on the circle of radius 1 and center at $(1,0)$. As the eigenvalues of $D^{\mathrm{FP}}$ lie very close to the GW circle, we see that $D^{\mathrm{FP}}$ satisfies the GW relation well.

The GW relation is equivalent to

$A^{\dagger} A=1, \quad A=1-D$.

In Fig. 2, we plot the 10 smallest eigenvalues of $A^{\dagger} A$ divided by the largest eigenvalue of $A^{\dagger} A$ for 10 Fixed-Point gauge configurations with lattice spacing $a \approx 0.13 \mathrm{fm}$. The largest eigenvalue is $\sim$ 1.5 and 38 for $D^{\mathrm{FP}}$ and $D^{\text {Wilson }}$ respectively. We see that the $A^{\dagger} A$ eigenvalues are much closer to 1 using $D^{\mathrm{FP}}$ than by using $D^{\mathrm{Wilson}}$. For larger volumes, $A^{\dagger} A$ is more likely to have a few very small eigenvalues, but the vast bulk of the eigenvalues is close to 1 .

An exact solution of the GW relation is given by the Overlap operator [9]

$D^{\text {Overlap }}=1-A / \sqrt{A^{\dagger} A}, \quad A=1-D_{0}$.

The already very good chiral behavior of $D^{\mathrm{FP}}$ can be made exact by using the Overlap construction. Very precise chiral symmetry is required for some measurements, such as the chiral condensate. Using $D^{\mathrm{FP}}, A^{\dagger} A$ is close to 1 and we use a polynomial approximation of $1 / \sqrt{A^{\dagger} A}$, which should converge rapidly. In Fig. 3 , we see that the breaking of the GW relation, given by

$B^{2}=\left|\left(D+D^{\dagger}-D^{\dagger} D\right) \cdot v\right|^{2}$,

where $v$ is a random vector, falls off exponentially as we increase the order of the polynomial approximation of $1 / \sqrt{A^{\dagger} A}$ and becomes $\leq \mathcal{O}\left(10^{-10}\right)$ at 


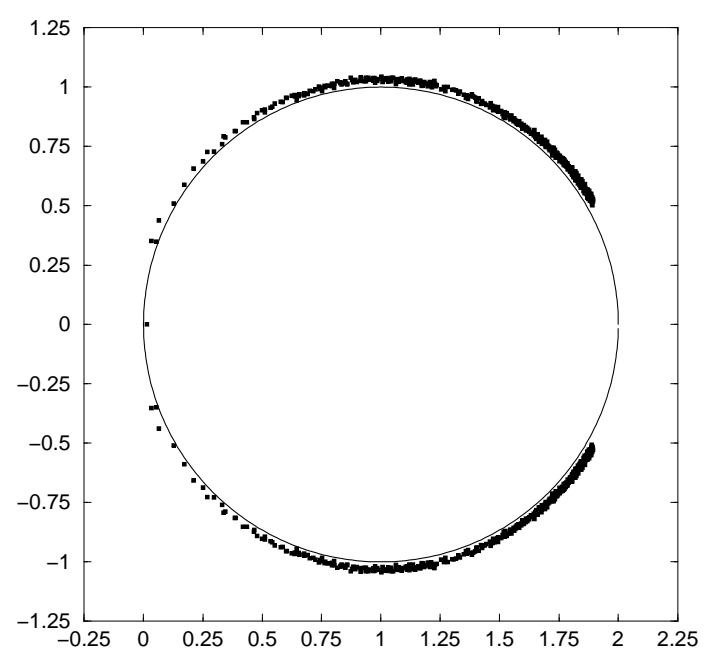

Figure 1. Eigenvalue spectrum of $D^{\mathrm{FP}}$.

polynomial order 10 for this volume. If the eigen-

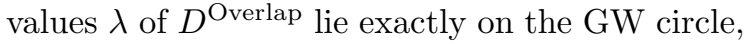
then $\Lambda=\lambda /(1-\lambda / 2)$ is purely imaginary. In Fig. 4 , we see that real $(\Lambda)$ falls off exponentially as we increase the polynomial order, as the eigenvalues $\lambda$ get closer and closer to the GW circle. The most rapid decrease is at the finest lattice spacing $a \approx 0.10 \mathrm{fm}$.

A lattice action must be local for the continuum results to be universal i.e. independent of the details of the lattice action. Locality means that fields at large separation have an exponentially small coupling. Optimizing the locality is essential so that e.g. the exponential fall-off of correlation functions can be separated from direct couplings in the lattice action. The locality of a lattice Dirac operator can be measured by

$f(r)=\stackrel{y}{\max }\{|D \cdot v|,\|y-x\|=r\}$,

where $v$ is a vector with point source at $x$ and $r$ is the square norm. In Fig. 5, we see that the Overlap operator constructed from the interacting $D^{\mathrm{FP}}$ is more local than by using the free $D^{\text {Wilson }}$, which in turn is more local than by using the interacting $D^{\text {Wilson }}$.

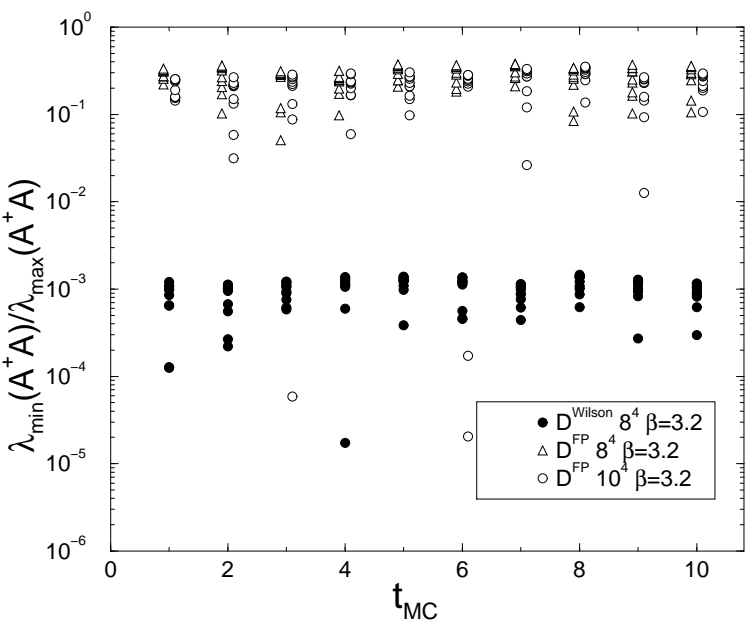

Figure 2. Chirality breaking of $D^{\text {Wilson }}$ and $D^{\mathrm{FP}}$.

\section{Chiral condensate}

For a theory of $N_{f} \geq 2$ massless quark flavors, the chiral symmetry is spontaneously broken if the chiral condensate $\langle\bar{\psi} \psi\rangle \neq 0$. Using chiral perturbation theory or random matrix theory, the chiral condensate at finite quark mass and volume has been calculated in the continuum, both for quenched and full QCD [10]. The quenched QCD condensate $\Sigma_{m, V, Q}=-\langle\psi \psi \psi\rangle_{m, V, Q}$ in topological sector $Q$ at small $m$ is

$\Sigma_{m, V, Q}=|Q| / m V+$

$m V \Sigma^{2}\left[I_{|Q|}(z) K_{|Q|}(z)+I_{|Q|+1}(z) K_{|Q|-1}(z)\right]$,

where $I_{Q}$ and $K_{Q}$ are modified Bessel functions, $z=m \Sigma V$ and $\Sigma=-\langle\bar{\psi} \psi\rangle$ is the quantity we want to measure. The term $|Q| / m V$ comes from the zero modes, whose contribution is not suppressed by the fermion determinant in quenched QCD at finite volume. Subtracting the contribution of the zero modes, for $Q \neq 0$

$\lim _{m \rightarrow 0} \frac{\Sigma_{m, V, Q}^{\mathrm{sub}}}{m}=\frac{\Sigma^{2} V}{2|Q|}$.

By measuring this ratio for several masses, volumes and topological sectors, the scaling behavior can be used to extract $\Sigma[11]$. 


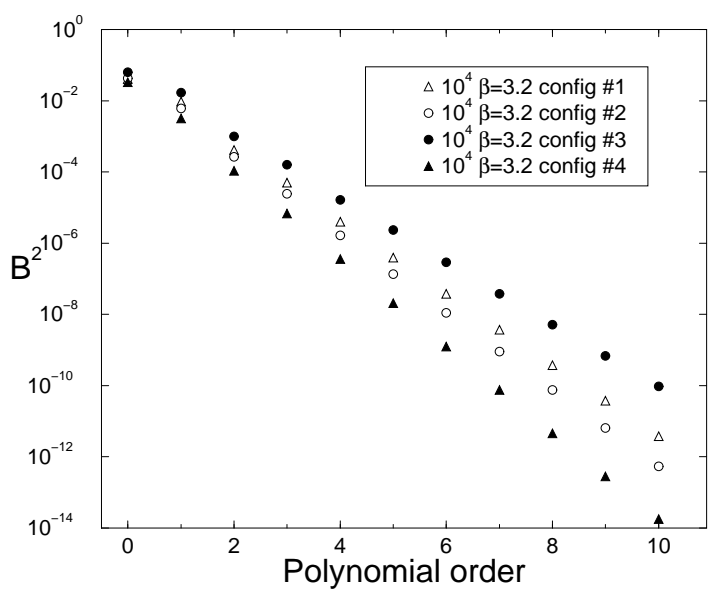

Figure 3. Breaking of Ginsparg-Wilson relation.

The subtracted condensate is measured by

$$
\begin{aligned}
& \sum_{m, V, Q}^{\mathrm{sub}}=\operatorname{Tr}^{\mathrm{sub}}\left[(D(m) 2 R)^{-1}-1 / 2\right] \\
& D(m) 2 R=(1-m / 2) D(0) 2 R+m,
\end{aligned}
$$

where $D$ and $R$ are related via the GW relation. We use the Overlap construction with $D^{\mathrm{FP}}$ (hence the Overlap $D^{\mathrm{FP}}$ ) so that the chiral symmetry is precise enough to go to quark mass $m a=10^{-4}$. The trace is measured stochastically using random $Z(2)$ vectors. The topology $Q$ is determined from the chirality of the lowest eigenmodes of $D^{\dagger} D$, found with an Arnoldi solver. For the topology scan, a Legendre polynomial of order 2 is used to approximate $1 / \sqrt{A^{\dagger} A}$. As a byproduct, we determine the topological susceptibility from an ensemble of $20010^{4}$ configurations to be $r_{0}^{4} \chi_{t}=0.0612(75)$ corresponding to $\chi_{t}=(196 \pm 6 \mathrm{MeV})^{4}$. In the volumes we examine, all $Q$ zero modes have the same chirality and their contribution to the condensate is subtracted by using random $Z(2)$ vectors whose chirality is opposite to those of the zero modes.

We measure the condensate in volumes $6^{4}, 8^{4}$ and $10^{4}$ in topological sectors $|Q|=1,2$ at lattice spacing $a \approx 0.13 \mathrm{fm}$. The statistics are given in Table 1 . We use 10 random $Z(2)$ vectors to measure the trace for each configuration and a multi-mass solver to invert $D$ simultaneously at a

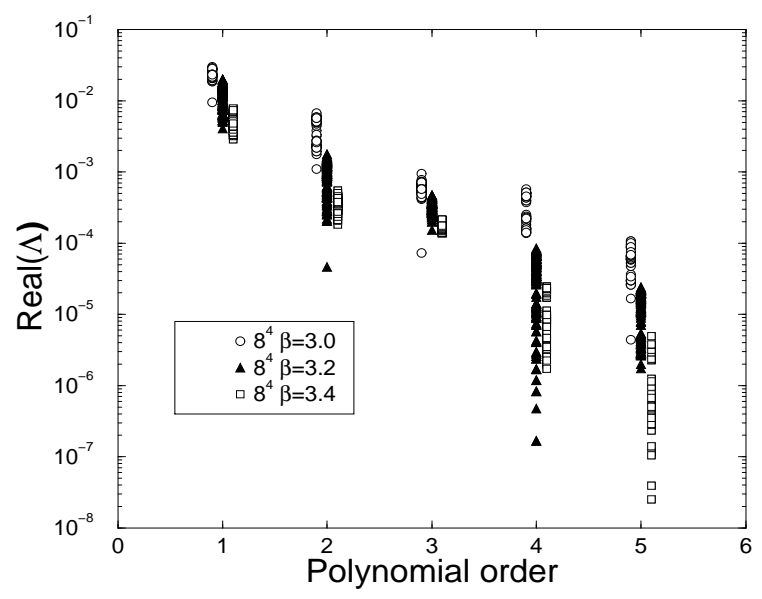

Figure 4. Deviation of $D^{\text {Overlap }}$ eigenvalues from GW circle.

number of masses. We use Legendre polynomials of order 5,7 and 10 to approximate $1 / \sqrt{A^{\dagger} A}$ for volumes $6^{4}, 8^{4}$ and $10^{4}$ respectively. This gives sufficiently precise chiral symmetry - increasing the polynomial order further, the relative change in $\Sigma_{m, V, Q}^{\text {sub }} / m$ is $\leq \mathcal{O}\left(10^{-4}\right)$. The 10 smallest $A^{\dagger} A$ eigenvalues are projected out and treated exactly. In Fig. 6, we plot $a^{3} \Sigma_{m, V, Q}^{\mathrm{sub}} / m a$ as a function of $m a$ for several volumes and topological sectors. We see that the data reach a plateau at small quark masses. Including lattice artifacts, the finite-size scaling behavior is

$\frac{\Sigma_{m, V, Q}^{\mathrm{sub}}}{m}=\frac{\Sigma^{2} V}{2|Q|}+\frac{c_{1}}{a^{2}}+\frac{c_{2} m}{a}+\ldots$,

where $c_{1}, c_{2}, \ldots$ are unknown coefficients which have to be fitted. It is natural to assume that these coefficients, dependent on the UV fluctuations, are independent of the topological charge $Q$. As shown in Fig. 7, we fit the data at $m a=$ $10^{-4}$ and determine that the bare condensate is $a^{3} \Sigma=4.68(26) \times 10^{-3}$. We can convert this using the Sommer parameter $\left(r_{0} / a=3.943(60)\right.$ has previously been measured at this bare coupling), which gives $r_{0}^{3} \Sigma=0.287(16)(13)$, where the first error is statistical and the second the uncertainty in the scale. 


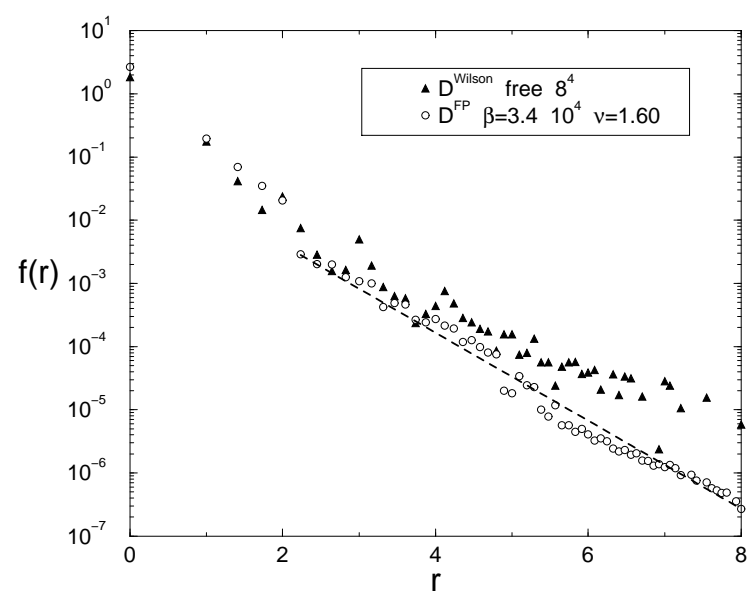

Figure 5. Locality of $D^{\text {Overlap }}$ using $D^{\text {Wilson }}$ and $D^{\mathrm{FP}}$ (exponential fall-off with $\nu \sim 1.6$ ).

The renormalization factor $Z_{S}$ for the chiral condensate is related to the quark mass renormalization by $Z_{S}=1 / Z_{m}$. The mass renormalization can be determined from hadron spectroscopy measurements, as done in [12]. Hadron mass measurements using the Overlap $D^{\mathrm{FP}}$ have been done at the same lattice spacing $a \approx 0.13 \mathrm{fm}$ [13], giv$\operatorname{ing} Z_{S}=0.80(6)$ in the renormalization group invariant scheme. Using this, the renormalized chiral condensate is $r_{0}^{3} \hat{\Sigma}=0.230(13)(10)(17)$, with statistical, scale and renormalization errors respectively. This gives $\hat{\Sigma}=(242 \pm 9 \mathrm{MeV})^{3}$, or in the $\overline{\mathrm{MS}}$ scheme, $\Sigma_{\overline{\mathrm{MS}}}(2 \mathrm{GeV})=(270 \pm 10 \mathrm{MeV})^{3}$. Comparing our measurement with other recent determinations of the renormalized chiral condensate [12,14], we see in Fig. 8 that there is good agreement.

\section{Local chirality of near zero modes}

From instanton physics, it is expected that near zero modes of a lattice Dirac operator are localized around instantons and anti-instantons and that, in these regions, the modes are predominantly either left- or right-handed. These near zero modes form a finite density $\rho(0)$ as $V \rightarrow \infty$, breaking the chiral symmetry via the

\begin{tabular}{|c|c|c|}
\hline$V$ & $|Q|$ & $N_{\text {conf }}$ \\
\hline \hline $6^{4}$ & 1 & 48 \\
\hline $8^{4}$ & 1 & 61 \\
\hline $8^{4}$ & 2 & 18 \\
\hline $10^{4}$ & 1 & 53 \\
\hline $10^{4}$ & 2 & 43 \\
\hline
\end{tabular}

Table 1

Statistics for the condensate measurement.

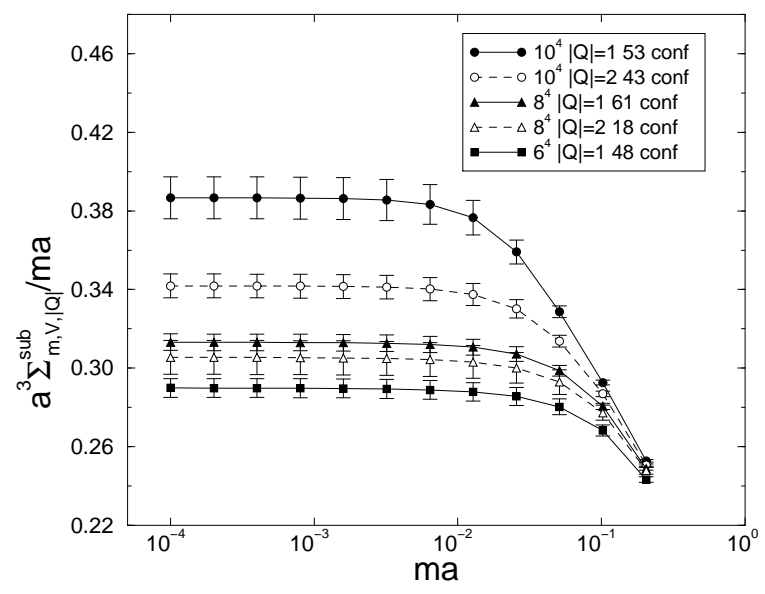

Figure 6. $a^{3} \Sigma_{m, V,|Q|} / m a$ vs. $m a$ for several volumes and topological sectors.

Banks-Casher relation $\langle\bar{\psi} \psi\rangle=-\pi \rho(0)$. A measure of the local chirality at lattice site $x$ is 15

$$
\tan \left[\frac{\pi}{4}(1+X(x))\right]=\sqrt{\frac{\psi_{\mathrm{L}}^{\dagger} \psi_{\mathrm{L}}(x)}{\psi_{\mathrm{R}}^{\dagger} \psi_{\mathrm{R}}(x)}} .
$$

If the near zero modes are localized and locally chiral, then where $\psi^{\dagger} \psi(x)$ is large, $X$ should be close to \pm 1 . If the modes are not locally chiral, then $X$ should be close to 0 .

We analyzed the 10 smallest near zero modes of the Overlap $D^{\mathrm{FP}}$ for $6010^{4}$ configurations at lattice spacing $a \approx 0.13 \mathrm{fm}$, using a Legendre polynomial of order 2 to approximate $1 / \sqrt{A^{\dagger} A}$ (these modes were already used to determine the topol- 


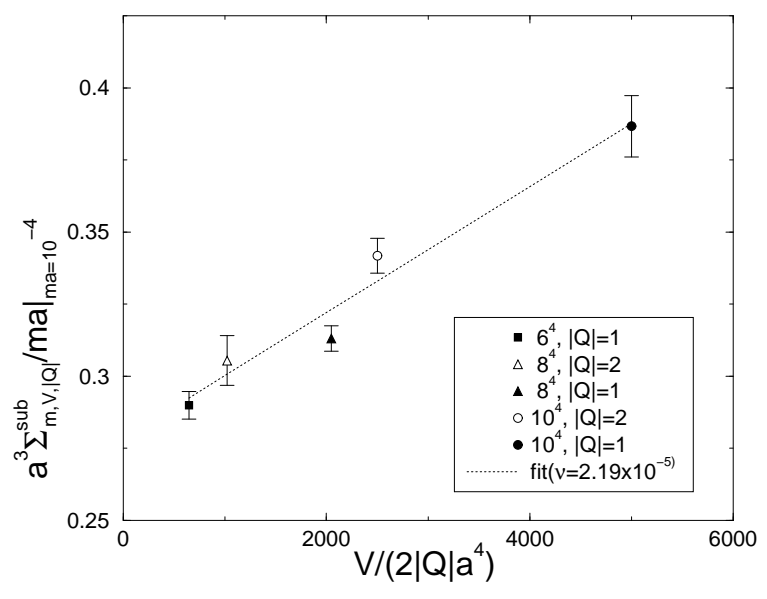

Figure 7. $\quad a^{3} \Sigma_{m, V,|Q|} / m a$ at $m a=10^{-4} \mathrm{vs}$. $V /\left(2|Q| a^{4}\right)$ and fit to finite-size scaling behavior.

ogy $Q$ of the gauge configurations). In Fig. 9, we plot the distribution $P(X)$, where we include the top $1 \%, 5 \%$ and $10 \%$ lattice sites $x$ where $\psi^{\dagger} \psi(x)$ is largest. We see a clear double-peaked distribution, with peaks far from $X=0$. Where the modes are most localized, they are also very chiral. This supports the picture of instanton dominance of the near zero modes, in agreement with [16, unlike the original claim of 15 .

\section{Construction of chiral currents}

For a chiral lattice action, the advantages of using the conserved currents are that $Z_{V}=Z_{A}=1$, the current and densities are automatically $\mathcal{O}(a)$ improved and expressions have the same form as in the continuum. The axial-vector current with the Overlap operator was already given in [17]. Here we define conserved currents (obtained via the Noether procedure) which are easy to use in numerical simulations. The vector and axialvector currents are

$V_{\mu}^{a}(x)=\bar{\psi} \tau^{a}\left[P_{L} K_{\mu}(x) \hat{P}_{R}+P_{R} K_{\mu}(x) \hat{P}_{L}\right] \psi$

$A_{\mu}^{a}(x)=\bar{\psi} \tau^{a}\left[P_{L} K_{\mu}(x) \hat{P}_{R}-P_{R} K_{\mu}(x) \hat{P}_{L}\right] \psi$

$P_{R, L}=\left(1 \pm \gamma_{5}\right) / 2 \quad \hat{P}_{L, R}=P_{L, R} \pm \gamma_{5} D / 2$. (11)

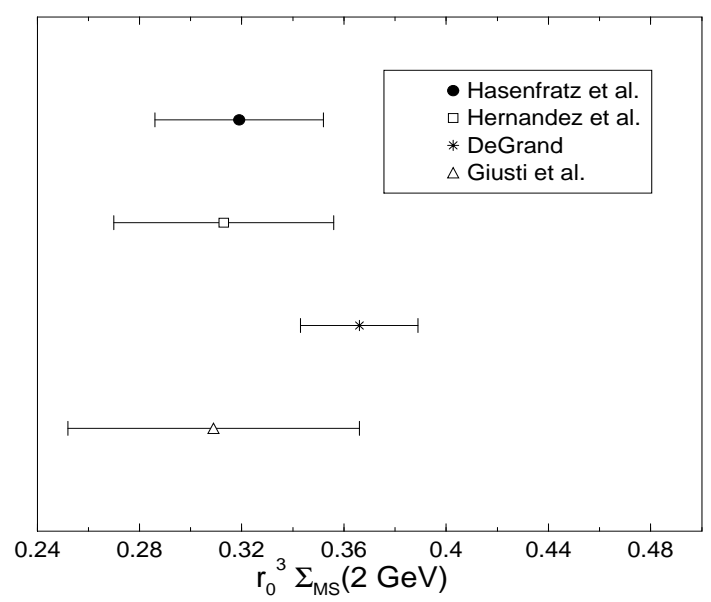

Figure 8. Comparison of measurements of $r_{0}^{3} \Sigma_{\overline{\mathrm{MS}}}(2 \mathrm{GeV})$ by different groups.

These currents are conserved and have the correct properties under global vector and axial-vector transformations e.g.

$$
\begin{aligned}
& i \frac{\delta}{\delta \epsilon^{a}} V_{\mu}^{b}(x)=i f^{a b c} A_{\mu}^{c}(x) \quad \text { axial-vector } \\
& i \frac{\delta}{\delta \epsilon^{a}} V_{\mu}^{b}(x)=i f^{a b c} V_{\mu}^{c}(x) \quad \text { vector }
\end{aligned}
$$

The kernel $K_{\mu}(x, U)$ which appears in the currents is defined by

$$
\begin{aligned}
K_{\mu}(x, U) & =-\left.i \frac{\delta D\left(\tilde{U}_{\mu}(x)\right)}{\delta \alpha_{\mu}(x)}\right|_{\alpha=0} \\
\tilde{U}_{\mu}(x) & =\mathrm{e}^{i \alpha_{\mu}(x)} U_{\mu}(x)
\end{aligned}
$$

The kernel can be calculated easily - the gauge link $U_{\mu}(x)$ receives an infinitesimal $U(1)$ rotation and the Dirac operator is calculated on this new and original gauge configuration (differing only at one link). The ratio of the difference of these two Dirac operators to the $U(1)$ variation gives $K_{\mu}(x, U)$. Details of the derivation and properties of the currents and densities will be given in [6]. 


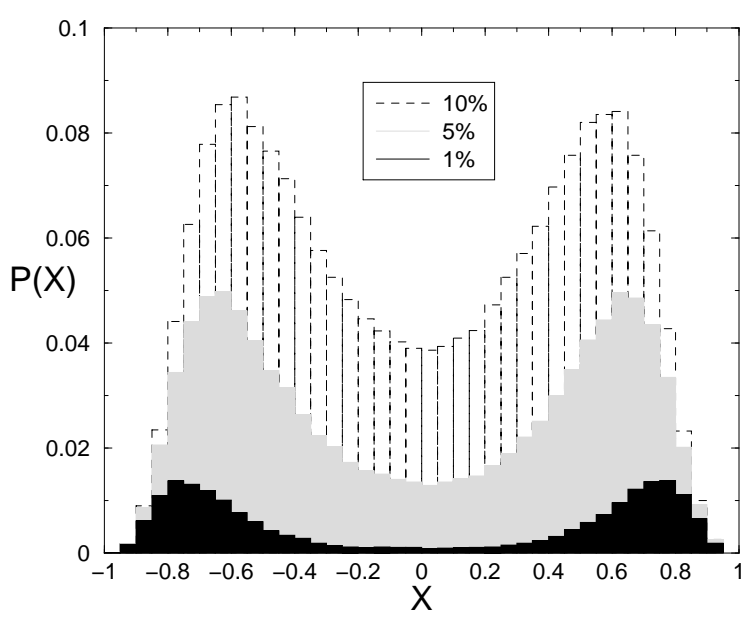

Figure 9. Distribution $P(X)$ for top $1 \%, 5 \%, 10 \%$ lattice sites $x$ with largest $\psi^{\dagger} \psi(x)$.

\section{Summary}

The parametrized Fixed-Point Dirac operator $D^{\mathrm{FP}}$ has very good chiral properties and loworder polynomial approximations can be used in the Overlap construction to make the chiral symmetry very precise. The Overlap operator is also much more local using $D^{\mathrm{FP}}$ than by using $D^{\text {Wilson }}$. Using finite-size scaling, the bare chiral condensate is measured as $r_{0}^{3} \Sigma=0.287(16)(13)$ and, estimating the renormalization factor from hadron spectroscopy, the renormalization group invariant condensate is $r_{0}^{3} \hat{\Sigma}=0.230(13)(10)(17)$, i.e. $\hat{\Sigma}=(242 \pm 9 \mathrm{MeV})^{3}$. In addition, the topological susceptibility is $\chi_{t}=(196 \pm 6 \mathrm{MeV})^{4}$. Near zero modes of the Dirac operator are locally chiral, which supports the picture that they are localized on instantons. We give a general and practical construction of chiral currents and densities, to be used in e.g. determining renormalization factors and decay constants.

\section{Acknowledgements}

This work is supported in part by the Schweizerischer Nationalfonds and the Eu- ropean Community's Human Potential Programme under contract HPRN-CT-2000-00145, Hadrons/Lattice QCD. We thank the Swiss Center for Scientific Computing in Manno, the University of Regensburg and LRZ München for computational resources.

\section{REFERENCES}

1. P. M. Vranas, Nucl. Phys. Proc. Suppl. 94 (2001) 177; P. Hernández, these proceedings.

2. P. Hasenfratz and F. Niedermayer, Nucl. Phys. B414 (1994) 785;

3. P. H. Ginsparg and K. G. Wilson, Phys. Rev. D25 (1982) 2649.

4. M. Lüscher, Phys. Lett. B428 (1998) 342.

5. P. Hasenfratz, Nucl. Phys. Proc. Suppl. 63 (1998) 53.

6. P. Hasenfratz et al., in preparation.

7. P. Hasenfratz et al., Nucl. Phys. Proc. Suppl. 94 (2001) 627; P. Hasenfratz et al., Int. J. Mod. Phys. C 12 (2001) 691.

8. F. Niedermayer, P. Rufenacht and U. Wenger, Nucl. Phys. B597 (2001) 413.

9. R. Narayanan and H. Neuberger, Phys. Rev. Lett. 71 (1993) 3251; Nucl. Phys. B412 (1994) 574; ibid. B443 (1995) 305; S. RandjbarDaemi and J. Strathdee, Phys. Lett. B348 (1995) 543; Nucl. Phys. B443 (1995) 386; ibid. B466 (1996) 335; Phys. Lett. B402 (1997) 134.

10. J. C. Osborn et al., Nucl. Phys. B540 (1999) 317; P. M. Damgaard et al., Nucl. Phys. B547 (1999) 305; H. Leutwyler and A. V. Smilga, Phys. Rev. D46 (1992) 5607.

11. P. Hernández et al., Phys. Lett. B469 (1999) 198.

12. P. Hernández et al., JHEP 0107 (2001) 018.

13. S. Hauswirth et al., hep-lat/0109004.

14. T. DeGrand, hep-lat/0107014; L. Giusti et al., hep-lat/0108007.

15. I. Horvath et al., hep-lat/0102003.

16. I. Hip et al., hep-lat/0105001; T. DeGrand and A. Hasenfratz, hep-lat/0103002; R. G. Edwards and U. Heller, hep-lat/0105004; T. Blum et al., hep-lat/0105006; C. Gattringer et al., hep-lat/0105023.

17. Y. Kikukawa and A. Yamada, Nucl. Phys. B547 (1999) 413. 